\title{
A Line Formula Notation System for Coordination Compounds: III. Deviations From Idealized Configurations
}

\author{
E. Silverton* and R. F. Pasternack**
}

(October 22, 1965)

\begin{abstract}
A linear notation describing the true configuration of a coordination complex has been developed to supplement the McDonnell-Pasternack representation of the idealized configuration. Criteria for determining major deviations from the ideal configuration are proposed. The symmetry of the true configuration is described with the aid of point-group notation; ligand sites are designated according to the system developed by McDonnell and Pasternack.
\end{abstract}

Key Words: Configuration, coordination, inorganic, linear notation, point-group, symmetry.

A previous study $[1,2]^{1}$ has led to a method of ciphering coordination complexes using a set of idealized structures. However, many important properties (spectra, magnetic susceptibility, etc.) of this class of compounds depend on the true symmetry of the species. Indeed, for certain group $\mathbf{M}$ atoms [1], the idealized structure is never closely approached in Nature. Cupric hexacoordinate complexes, for example, never appear octahedral, but always show large distortions. It is therefore appropriate to include the true configuration in the store of information about the given substance.

The idealized structure is represented in File I by the McDonnell-Pasternack cipher and the true configuration (if it differs from the ideal) is described in File II in terms of its point group notation [3]. As the transition from the ideal to the nonideal configuration is a gradual one, a criterion is set to distinguish the so-called "minor" deviations from the "major" deviations. A deviation is termed major when it cannot be attributed to inaccuracies in atomic parameters. If the apparent deviation of a bond length or angle is 2.3 times as large as the corresponding estimated standard deviation (e.s.d.) ${ }^{2}$ the deviation is taken to be real [5]. The e.s.d. thus serves as a criterion for classifying apparent deviations. When all deviations are minor, the structure is treated ideally, and the symbol "\#" is stored in File II. File II is omitted when the structural information ${ }^{3}$ given is insufficient to derive deviations.

The accuracy of structure determinations reported in the literature covers a wide range, although the trend is to more accurate determinations. This presents a problem in the consideration of deviations: a deviation classified as a minor in a relatively inaccurate determination can be major in an accurately determined structure. Therefore, if no major devi-

*Present address: National Institutes of Health, Bethesda, Md.

**Present address: Department of Chemistry, Ithaca College, Ithaca, New York.

${ }^{1}$ Figures in brackets indicate the literature references at the end of this paper.

${ }^{2}$ The e.s.d. measures the precision of bond distances and angles. See reference [4].

${ }^{3}$ For the purposes of this paper, the structural data are taken from crystallographic studies. ations are disclosed in a structure, does this indicate a close approach to ideality or merely reflect a low degree of accuracy in the structure determination? To resolve this question it seems appropriate to grade the quality of a structure determination according to its minimum significant deviation from ideality. As the classification of deviations is based upon e.s.d., this becomes a natural criterion in assigning the abovedescribed grades. The proposed grades are in table 1 .

TABLE 1. Proposed grades

\begin{tabular}{c|c|c}
\hline \hline Grade & e.s.d. of Ligand-metal bond & e.s.d. of Ligand-metal-ligand \\
\hline & $(\AA)$ & Angle (deg $)$ \\
A & $\leq 0.01$ & $\leq 0.3$ \\
B & $0.01-0.03$ & $0.3-0.6$ \\
C & $0.03-0.05$ & $0.6-0.7$ \\
D & $>0.05$ & $>0.7$ \\
& and all two-dimensional structure determinations. \\
\hline
\end{tabular}

The point group of the true configuration contains only those symmetry elements of the idealized configuration from which there are no major deviations. Therefore, the first step in determining the true configuration is to test the symmetry elements of the idealized configuration for deviations. Ligands are treated as identical spheres (bidentates are represented by two spheres, tridentates by three, etc.) in determining symmetry elements. Three types of symmetry elements are used in point-group notation: inversion center, rotation axis, and reflection plane (mirror plane). Combinations of these symmetry elements produce additional symmetry elements: rotoreflection axis (alternating axis) and rotoinversion axis (inversion axis). An $n$-fold axis of rotation rotates each atom $360^{\circ} / n$ about the axis, generating $\mathrm{N}$ atoms in a plane perpendicular to the axis. Therefore the conditions for an $n$-fold are (1) the $n$ atoms display the axial symmetry, (2) the $n$ atoms are coplanar, (3) the plane of the $n$ atoms is perpendicular to the rotation axis. Deviations from these conditions may be expressed in different ways. The following are possible criteria 
for testing axial symmetry: (1) the distances of the $n$ atoms from the axis of rotation, (2) the interatomic distances between adjacent planar atoms, and (3) the angle subtended by adjacent planar atoms and the rotation axis. The e.s.d. of the corresponding bond distance or angle is used in classifying possible deviations from the average value. Two ways of expressing degree of coplanarity are (1) distances of the atoms from the mean plane, and (2) the sum of the interior angles in the polygon formed by the $n$ atoms. In the first case, the apparent deviation is compared with the e.s.d. of the atomic coordinates and the e.s.d. of the angles are used in the second case.

Any molecule with an atom lying on a symmetry element of the space group ${ }^{4}$ will contain that symmetry element. (These space-group positions are called special positions.) Thus, if an atom in a coordination complex occupies a special position, the true configuration of the coordination complex will contain the symmetry element corresponding to the special position.

After the point group of the true configuration is determined, a rotational axis is selected according to the following criteria:

\section{The highest-order axis.}

2. The axis coinciding with the highest-order axis of the idealized structure.

3. The axis containing the earliest locant-designator.

4. The axis perpendicular to the plane containing the earliest locant-designator.

The senior end of the axis is the one passing through the most senior (earliest) locant position (or the plane containing the most senior locant position) at first difference.

The cipher describing the true configuration makes use of the enumeration pattern and locant designation given in File I [1]. In the idealized configuration the assignment of ligands to locant sites may be arbitrary for identical ligands occupying positions in a plane equidistant from the axis or occupying equivalent positions on the axis. However, if distortions from the idealized configuration exist, these ligand sites may no longer be equivalent. In this case ligands are assigned locants in order of increasing distance from the axis of rotation (if they lie in a plane) or from the group $M$ atom (if they lie on the axis). The File II cipher begins with the Schoenflies notation [7,3] for the point group of the true configuration, followed by a comma. The order $(2,3,4$, etc.) of the selected rotational axis is cited next, followed by an opening brace. Atoms are cited as they appear along the axis (starting from the senior end), separating atoms at different levels by slashes. Atoms not on the axis and equidistant from the axis, lying in a plane perpendicular to the axis, are separated by commas and enclosed within parentheses. A period precedes the

${ }^{4}$ The 230 space groups describe all combinations of symmetry elements possible in crystals. See reference [6]. opening parenthesis. If another set of atoms lies in the same plane at a greater distance from the axis, the sets are cited in order of increasing distance and are separated by periods. Atoms within parentheses are cited (1) in numerical order, ${ }^{5}$ and (2) in alphabetic order of the locants. If an axial atom lies on the above described plane, it is cited before the initial period. If a single nonaxial atom occurs, it is preceded by a period. The cipher is completed by a closing brace, followed by the grade.

The following examples illustrate various degrees of deviation from the ideal configuration as well as the use of the criteria for testing symmetry elements.

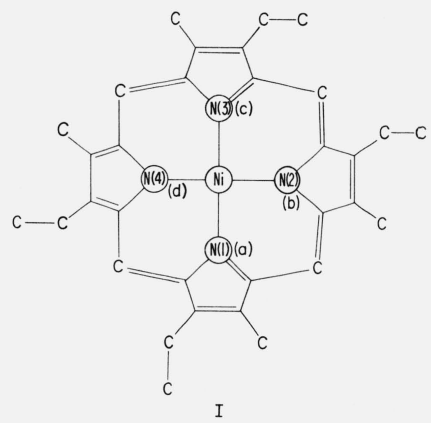

EXAMPLE I. Nickel Etioprophyrin II [8] $\mathrm{NiC}_{32} \mathrm{H}_{36} \mathrm{~N}_{4}$ (I)

Type: square planar (point group $\mathrm{D}_{4 \mathrm{~h}}$ ).

Special positions: $\mathrm{Ni}$ at $\overline{4} \mathrm{M} 2, \mathrm{~N}$ on a mirror plane and $\mathrm{C}$ on a 2 -fold axis.

Grade: D.

File I cipher ${ }^{6}$ : ni $\{1,4 \mathrm{~B}\} \ll 5 \mathrm{~L}(\mathrm{~L}=@ 5 \mathrm{LN}[1 \mathrm{a}] \mathrm{L}($

$$
\begin{aligned}
& =\mathrm{L} @ 5 \mathrm{~L}=\mathrm{N}[1 \mathrm{~b}] \mathrm{L}(=\mathrm{L} @ 5 \mathrm{LN}[1 \mathrm{c}] \\
& \mathrm{L}(\mathrm{L}=@[1])=\mathrm{L}(\mathrm{CM}) \mathrm{LM}=) \mathrm{LM} \\
& \quad=\mathrm{L}(\mathrm{CM}) \mathrm{L}(\mathrm{CM})=\mathrm{LM}) \\
& \quad=\mathrm{N}[1 \mathrm{~d}] \mathrm{L}[1] \mathrm{L}(\mathrm{CM})=\mathrm{LM} .
\end{aligned}
$$

The square planar configuration contains the following combination of symmetry elements: a 4 -fold axis with four 2-fold axes and a mirror plane perpendicular to it. The conditions for a 4-fold axis through the nickel atom are that the four nitrogen atoms (1) be equidistant from the nickel, (2) subtend $90^{\circ}$ angles with the nickel, and (3) be coplanar with the nickel atom. Note that the third condition also satisfies the requirement for a mirror plane perpendicular to the 4 -fold axis. Since the nitrogen atoms lie on two perpendicular mirror planes intersecting at the nickel atom, the first two conditions are satisfied. The third condition will be discussed later.

\footnotetext{
${ }^{5} \mathrm{~A}$ number referring to the coordination center is used if more than one coordination center exists.

${ }^{6}$ The square planar configuration is referred to as $4 \mathrm{~B}$ in the File $\mathrm{I}$ eipher. See reference [1].
} 
The four potential 2 -fold axes are as follows:

(1) An axis bisecting angles $\mathrm{N}(1) \mathrm{NiN}(2)$ and $\mathrm{N}(3) \mathrm{NiN}(4)$,

(2) an axis bisecting angles $\mathrm{N}(2) \mathrm{NiN}(3)$ and $\mathrm{N}(1) \mathrm{NiN}(4)$,

(3) an axis through $\mathrm{N}(1), \mathrm{N}(3)$, and $\mathrm{Ni}$,

(4) an axis through $\mathrm{N}(2), \mathrm{N}(4)$, and Ni.

The first two axes are the ones on which the carbon atoms lie. If the third axis exists, $\mathrm{N}(2)$ and $\mathrm{N}(4)$ must be related by 2 -fold symmetry. This is true if $\mathrm{N}(2)$ and $\mathrm{N}(4)$ are coplanar with $\mathrm{N}(1), \mathrm{N}(2)$, and Ni. Similarly this condition also satisfies the requirements for the fourth axis. A look at the atomic coordinates of nitrogen and nickel shows that the condition would be met if the $z$-coordinate of nitrogen were 0.125 . The $z$-coordinate is 0.122 , a deviation of 0.003 from the ideal. As the standard deviation of the $z$-coordinate is approximately 0.002 , the deviation is minor. ${ }^{7}$ Since there are no deviations from the ideal configuration, File II contains "\#, D" where D is the grade.

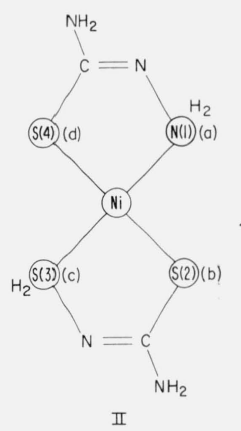

EXAMPLE II. Bis(thiosemicarbazidato)nickel(II)[9] $\mathrm{Ni}\left[\mathrm{SC}\left(\mathrm{NH}_{2}\right)=\mathrm{NNH}_{2}\right]_{2}(\mathrm{II})$

Type: square planar (point group $\mathrm{D}_{4 \mathrm{~h}}$ ).

Special positions: Ni on center of inversion.

Grade B

File I cipher: $\quad \operatorname{ni}\{1,4 \mathrm{~B}\} \ll \mathrm{C}(\mathrm{S}[1 \mathrm{~b}, 1 \mathrm{~d}]) \mathrm{Z}(=\mathrm{NZ}[1 \mathrm{a}$, lc]) $\gg 2$.

The point group $\mathrm{D}_{4 \mathrm{~h}}$ contains a 4 -fold axis with four 2 -fold axes and a mirror plane perpendicular to it.

The conditions for a 4 -fold axis through the nickel atom are that the ligand atom to nickel distances be equal (see table 2,a) and the angles subtended by adjacent ligands and the nickel atom be $90^{\circ}$ (see table $2, \mathrm{~b})$.

The deviations from 4-fold symmetry are major.

\footnotetext{
${ }^{7}$ The apparent deviation is less than 2.3 times the e.s.d.
}

TABLE 2 Bond parameters

\begin{tabular}{ll|l|l}
\hline \hline & & Deviations & e.s.d. \\
\hline a $\quad \mathrm{NiS}, \mathrm{NiN} \quad 2.155,1.911 \AA$ & & $0.122 \AA$ & $0.014 \AA$ \\
b & $\mathrm{N}(1) \mathrm{NiS}(4), \mathrm{N}(1) \mathrm{NiS}(2) \quad 81.7,98.3^{\circ}$ & $8.3^{\circ}$ & $.4^{\circ}$ \\
\hline
\end{tabular}

There are five possible 2-fold axes:

(1) An axis through the nickel atom perpendicular to the plane of the ligands.

(2) An axis through the two nitrogens and the nickel atom.

(3) An axis through the two sulfurs and the nickel atom.

(4) \& (5) Two axes bisecting the N-Ni-S angles.

Axis (1) is the only one having no major deviation. Axes (2) and (3) show major deviations from the required N-Ni-S angles. Axes (4) and (5) show major deviations in the ligand to metal distances.

The special position of the nickel atom requires that it be coplanar with the four ligands. Thus the symmetry elements of the true configuration are a 2 -fold axis with a mirror plane perpendicular to it; that is, $\mathrm{C}_{2 \mathrm{~h}}$. File II is written:

$$
\mathrm{C}_{2 \mathrm{~h}}, 2\{\mathrm{l} \cdot(\mathrm{a}, \mathrm{c}) \cdot(\mathrm{b}, \mathrm{d})\} \mathrm{B} .
$$

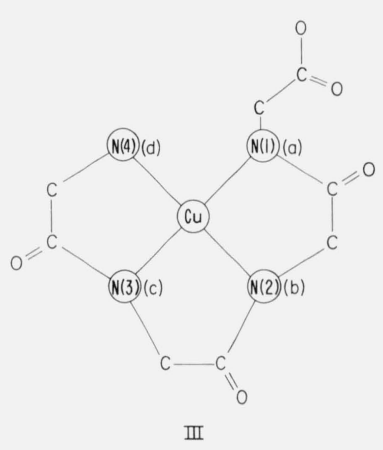

Example III. Disodium $N$-[N-(N-glycylglycyl) glycyl]glycinocuprate(II) decahydrate[10] $\mathrm{Na}_{2}\left[\mathrm{CuNH}{ }_{2}\right.$ $\left.\left(\mathrm{CH}_{2} \mathrm{CON}\right)_{3} \mathrm{CH}_{2} \mathrm{COO}\right] \cdot 10 \mathrm{H}_{2} \mathrm{O}$ (III)

Type: square planar (point group $\mathrm{D}_{4 \mathrm{~h}}$ ).

Special positions: None.

Grade: A.

File I cipher: $[\mathrm{cu}\{1,4 \mathrm{~B}\} \ll \mathrm{C}(\mathrm{O}) \mathrm{V}\{\mathrm{CN}[\mathrm{la}, \mathrm{lb}, 1 \mathrm{c}] \mathrm{CV}\}$ $3 \mathrm{CZ}[1 \mathrm{~d}] \gg] \Theta 2$.

Again the configuration is square planar, but in this case no atoms occupy special positions and so all symmetry elements must be tested.

The potential 4-fold axis passes through the copper atom perpendicular to the plane of the nitrogen atoms. Therefore the four ligands should be equidistant from the copper atom (see table $3 \mathrm{a}, \mathrm{a}, \mathrm{b}, \mathrm{c}, \mathrm{d}$ ) and subtend equal angles with the axis (see table $3 \mathrm{~b}, \mathrm{e}, \mathrm{f}, \mathrm{g}, \mathrm{h}$ ).

The deviations from 4-fold symmetry are major. The five potential 2 -fold axes which have been described in Example II all show major deviations in this case. Axes (1) and (3) require that $\mathrm{CuN}(4)=\mathrm{CuN}(2)$. Axis (2) requires that the angles subtended by adjacent ligands and the copper atom be $90^{\circ}$. The conditions for axes (4) and (5) include $\mathrm{CuN}(4)=\mathrm{CuN}(3)$ and $\mathrm{CuN}(1)=\mathrm{CuN}(4)$, respectively. 
TABle 3a. Distances ( $\mathrm{A})$

\begin{tabular}{c|c|c|c|c}
\hline \hline & & & $\begin{array}{c}\text { Deviations } \\
\text { from average }\end{array}$ & e.s.d. \\
\cline { 4 - 5 } & & & $\AA$ & $\AA$ \\
$\mathrm{a}$ & $\mathrm{CuN}(4)$ & 2.028 & 0.08 & 0.005 \\
$\mathrm{~b}$ & $\mathrm{CuN}(3)$ & 1.923 & .03 & .004 \\
$\mathrm{c}$ & $\mathrm{CuN}(2)$ & 1.912 & .04 & .004 \\
$\mathrm{~d}$ & $\mathrm{CuN}(1)$ & 1.944 & .01 & .004 \\
\hline
\end{tabular}

TABLE 3b. Angles (degrees)

\begin{tabular}{c|c|c|c|c}
\hline \hline & & $\begin{array}{c}\text { Deviations } \\
\text { from average }\end{array}$ & e.s.d.i \\
\cline { 3 - 4 } & & & Degrees & Degrees \\
& & & 6.5 & 0.2 \\
$\mathrm{e}$ & $\mathrm{N}(3) \mathrm{CuN}(4)$ & 83.5 & .2 \\
$\mathrm{f}$ & $\mathrm{N}(1) \mathrm{CuN}(4)$ & 109.8 & 19.8 & .2 \\
$\mathrm{~g}$ & $\mathrm{~N}(1) \mathrm{CuN}(2)$ & 84.0 & 6.0 & .2 \\
$\mathrm{~h}$ & $\mathrm{~N}(3) \mathrm{CuN}(2)$ & 82.8 & 7.2 & .2 \\
\hline
\end{tabular}

The remaining possible symmetry element is a mirror plane through the copper and the four ligands. Two criteria for planarity are (1) the sum of the angles subtended by adjacent ligands and the copper atom and (2) the deviations from the mean plane. The sum of the angles is $360.1^{\circ}$, indicating a minor deviation. The deviations from the mean plane are listed in table 4.

The deviations from the mean plane are major. This contradiction between the two criteria is not unexpected as the second is more sensitive to small deviations.

File II is written:

$$
\mathrm{C}_{1}, 1\{\mathrm{l} \cdot \mathrm{b} \cdot \mathrm{c} \cdot \mathrm{a} \cdot \mathrm{d}\} \mathrm{A} .
$$

TABLE 4 Deviations from mean plane

\begin{tabular}{l|r|r|r}
\hline \hline & Atom & Deviation & e.s.d. \\
\hline & & $\AA$ & $\AA$ \\
a & $\mathrm{Cu}$ & \multicolumn{1}{|c}{$\AA$} & $\AA$ \\
$\mathrm{b}$ & $\mathrm{N}(4)$ & .029 & 0.009 \\
$\mathrm{c}$ & $\mathrm{N}(3)$ & .046 & .017 \\
$\mathrm{~d}$ & $\mathrm{~N}(2)$ & .040 & .017 \\
$\mathrm{e}$ & $\mathrm{N}(1)$ & .039 & .017 \\
\hline
\end{tabular}

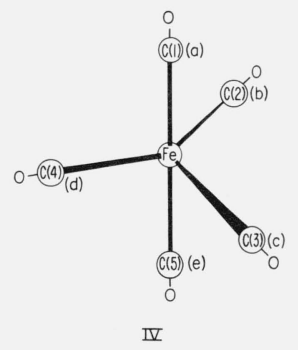

The point group $D_{3 \mathrm{~h}}$ consists of a 3 -fold axis with three 2-fold axes and a mirror plane perpendicular to it. The potential 3-fold axis passes through $\mathrm{C}(1), \mathrm{C}(5)$, and $\mathrm{Fe}$ perpendicular to the plane of ligands $\mathrm{C}(2), \mathrm{C}(3)$, and $\mathrm{C}(4)$. The conditions for 3-fold symmetry are (1) that ligands $C(2), C(3)$, and $C(4)$ be equidistant from the iron atom, and (2) that each angle subtended by any two of these ligands and the iron atom be 120. An examination of the parameters listed in table 5a shows a major deviation (parameter e) from the above conditions. The three possible 2-fold axes are (1) an axis through $\mathrm{C}(2)$ bisecting the angle subtended by ligands C(3) and C(4) and the iron atom, (2) an axis through C(3) bisecting the angle subtended by ligands $C(2)$ and $C(4)$ and the iron atom, and (3) an axis through C(4) bisecting the angle subtended by ligands $C(2)$ and $C(3)$ and the iron atom. The first 2 -fold axis need not be tested for deviations since the iron atom and ligand C(2) lie on a 2 -fold axis of the space group (i.e., a special position). The following conditions are required by the second and third 2fold axes respectively:

$$
\begin{aligned}
& \mathrm{C}(2) \mathrm{FeC}(3)=\mathrm{C}(3) \mathrm{FeC}(4) \\
& \mathrm{C}(2) \mathrm{FeC}(4)=\mathrm{C}(3) \mathrm{FeC}(4)
\end{aligned}
$$

Inspection of table $5 \mathrm{~b}$ reveals major deviations from these conditions. The final symmetry element of $\mathrm{D}_{3 \mathrm{~h}}$ to be tested is a mirror plane containing ligands $\mathrm{C}(2), \mathrm{C}(3)$, and $\mathrm{C}(4)$ and the iron atom. The conditions for the mirror plane are (1) that ligands $\mathrm{C}(1)$ and $\mathrm{C}(5)$ 
be equidistant from the mirror plane ${ }^{8}(2)$ that the line connecting ligands $\mathrm{C}(1)$ and $\mathrm{C}(5)$ be perpendicular to the mirror plane, (see table $5 \mathrm{~b}, \mathrm{~g}-\mathrm{j}$ ) and (3) that the sum of the angles subtended by ligands $\mathrm{C}(2), \mathrm{C}(3)$, and $\mathrm{C}(4)$ and the iron atom be $360^{\circ}$ (see table $5 \mathrm{~b}$, $\mathrm{d}, \mathrm{e}, \mathrm{f})$. Therefore, the mirror plane is a valid symmetry element. The point group of the true configuration is $\mathrm{C}_{2 \mathrm{v}}$. File II is written:

$$
\mathrm{C}_{2_{\mathrm{v}}}, 2\{\mathrm{~b} / \mathrm{l} \cdot(\mathrm{a}, \mathrm{e}) / \cdot(\mathrm{c}, \mathrm{d})\} \mathrm{D} \text {. }
$$

\section{References}

[1] P. M. McDonnell and R. F. Pasternack, J. Chem. Doc. 5, 56 (1965).

[2] R. F. Pasternack and P. M. McDonnell, Inorg. Chem. 4, 600 (1965).

${ }^{8} \mathrm{C}(1)$ and $\mathrm{C}(5)$ are related by the 2 -fold axis, which lies in the mirror plane, and are therefore equidistant from the plane.
[3] F. A. Cotton, Chemical Applications of Group Theory, pp. 38-39 (Interscience Publishers, New York, N.Y., 1963).

[4] D. W. J. Cruickshank, Acta Cryst. 2, 65 (1949).

[5] H. Lipson and W. Cochran, The Determination of Crystal Structures. The Crystalline State, Vol. III, p. 309 (G. Bell and Sons, London, 1957).

[6] International Tables for X-ray Crystallography, Vol. I (The Kynoch Press, Birmingham, England, 1952).

[7] A. Schoenflies, Krystallsysteme und Krystallstructur, Leipzig, 1891.

[8] M. B. Crute, Acta. Cryst. 12, 24 (1959).

[9] L. Cavalca, M. Nardelli, and G. Fava, Acta Cryst. 15, 1139 (1962).

[10] H. C. Freeman and M. R. Taylor, Acta Cryst. 18, 939 (1965).

[11] J. Donohue and A. Caron, Acta Cryst. 17, 663 (1964).

(Paper 70A1-384) 